\title{
Diálogo entre reconocimiento y desarrollo
}

\section{Dialogue between recognition and development}

Carolina Godoy-Berthet (c.godoy11@ufromail.cl) Núcleo Científico y Tecnológico de Ciencias Sociales, Universidad de la Frontera (Temuco, Chile) ORCID: 0000-0003-3460-0250

Mauricio Alarcón-Silva (m.alarcon11@ufromail.cl) Núcleo Científico y Tecnológico de Ciencias Sociales, Universidad de la Frontera (Temuco, Chile) ORCID: 0000-0002-7234-0392

\begin{abstract}
This article aims to establish a dialogue between notions of development and recognition. The epistemic foundation of this discussion is framed in Ricoeur consideration that bets on the individual's condition as a capable man or "I can", with the power to speak, act and impute actions, basic conditions to face the path to recognition of himself. However, these individual capacities to act, on the collective level, extend to social practices of mutual recognition based on an ethical principle, from which, it is possible to identify three imperatives dialoguing with the notion of development: to tend towards the good life, in and with others, and with just institutions. It concludes by affirming that the crisis regarding the notion of development proper to modernity has given it the possibility of resignifying the approaches that exalt human capacities in the search for subjective well-being that are oriented towards the path of mutual recognition.
\end{abstract}

Key words: recognition, social practices, development, ethics, modernity.

\section{Resumen}

El presente artículo se propone establecer un dialogo entre las nociones de desarrollo y de reconocimiento. La fundamentación epistémica de esta discusión se enmarca en la consideración de Ricoeur que apuesta a la condición del individuo como un hombre capaz o "un yo puedo", con el poder de hablar, actuar e imputarse acciones, condiciones básicas para enfrentar el camino hacia el reconocimiento de sí mismo. Ahora bien, dichas capacidades individuales de obrar, en el plano colectivo se extienden hacia prácticas sociales de reconocimiento mutuo sustentadas bajo un principio ético, desde el cual, es posible identificar tres imperativos dialogantes con la noción de desarrollo: tender hacia la vida buena, en y con el otro y con instituciones justas. Se concluye afirmando que la crisis respecto de la noción de desarrollo propia de la modernidad ha dado a la misma la posibilidad de resignificar los enfoques que exaltan las capacidades humanas en la búsqueda de un bienestar subjetivo que se orienten hacia el camino del reconocimiento mutuo.

Palabras clave: reconocimiento, prácticas sociales, desarrollo, ética, modernidad. 


\section{Introducción}

La noción de desarrollo ampliamente internalizada en el sentido común de la sociedad occidental asocia su significado a la idea de bienestar y progreso. Su uso moderno se vincula al esfuerzo deliberado de reorganización mundial de la segunda posguerra, cuyo hito más recordado es el discurso del presidente de Estados Unidos Harry Truman el año 1949, quien hace un llamado mundial a iniciar un camino hacia la construcción de sociedades avanzadas, esto es con "altos niveles de industrialización y urbanización, tecnificación de la agricultura, rápido crecimiento de la producción material y los niveles de vida, y adopción generalizada de la educación y los valores culturales modernos" (Escobar 2007:20).

A partir de entonces, el desarrollo como meta relato de la modernidad deja su impronta en la construcción de un tipo de subjetividad amparada y movilizada por el capitalismo global. Al respecto, Quintero propone dos senderos analíticos para desentrañar con profundidad el concepto de desarrollo: "por una parte, se requiere de la exploración de la constitución histórica del desarrollo como una formación subjetiva moderna; y, por otro lado, pero de manera simultánea, es menester la indagación del mismo como una expresión integral del capitalismo global" (Quintero 2013:69).

Las condiciones históricas, imbricadas en los ideales de la ilustración, sustentaron una construcción de imaginarios sociales referidos a territorios y grupos sociales específicos, ubicados aún en niveles inferiores del proceso civilizatorio. En tal sentido, la construcción de la subjetividad moderna se constituye a la vez en un instrumento de clasificación social que ubica por un lado al hombre ilustrado, moderno y europeo y, por otro, al hombre incivilizado, inferior y ubicado geopolíticamente en territorios conquistados. Al respecto Quintero plantea que "la inauguración de esta nueva conciencia histórica es la que otorga significación a la idea de desarrollo, que será, al mismo tiempo, uno de los ejes centrales de esta nueva conciencia" (2013:71).

En este sentido, la noción de desarrollo es coherente con un imaginario social del tiempo, propio de la racionalidad moderna, que sitúa el futuro como reino de la esperanza por "la edad dorada por conquistar o construir" (Quijano 1988:12). Se trata de la expectativa de un estado de situación que debe mejorar, que avanza temporalmente de manera continua hacia una condición deseada que ubica el bienestar como fin último, quedando en la incertidumbre o al arbitrio ideológico-burocrático respuestas a interrogantes éticas tales como: ¿quién es sujeto de ese bienestar?, ¿en qué consiste ese bienestar?, ¿qué debe ser valorado como bienestar?, ¿quién define ese bienestar?, ¿cómo se alcanza?, ¿bajo qué estrategia? Dichas preguntas son parte de los denominados determinantes (cooperación para) que posibilitan la operatividad de la noción de desarrollo (Jiménez Castillo. Una arqueología hegeliana de las teorías del desarrollo).

Ahora bien, desde una mirada normativa se asume la existencia de un sesgo ontológico que impide conciliar las pretensiones de imparcialidad y pluralismo de las teorías del desarrollo, por tanto, una imposibilidad empírica de un desarrollo en términos absolutos, pues sería una cuestión que impediría responder acabadamente las anteriores preguntas (Jiménez Castillo. Una arqueología hegeliana de las teorías del desarrollo). Sin embargo, "esto último no le arrebata relevancia, pues su facultad inmanente reside en iluminar acerca de su propio 'hacerse', es decir, el intento nunca conseguido de reconciliar el hiato entre imparcialismo y pluralismo es lo que, a fin de cuentas, estimula el perfeccionarse de una teoría del desarrollo frente a la otra" (Jiménez Castillo 2017:187).

Bajo este interés, planteamos una reflexión en torno a dichas interrogantes mediante los caminos del reconocimiento propuestos por Ricoeur. Centraremos nuestra discusión respecto de las capacidades del 
hombre y del rol de la alteridad en el reconocimiento de sí mismo, como base para ir ampliando la discusión hacia las capacidades sociales y de obrar de agentes que se comprometen en sus acciones, para finalmente proceder a interrogar la noción de desarrollo en el contexto de modernidad.

\section{La modernidad como promesa de emancipación del hombre}

La idea de emancipación del hombre como producto de la modernidad se asocia al concepto de llustración de Immanuel Kant, quien en un texto publicado el año 1784 denominado Respuesta a la pregunta ¿qué es Ilustración? la caracteriza como la salida del hombre de su condición de menor de edad de la cual él mismo es culpable. Luego, relaciona el estado de minoría de edad con una voluntaria condición de pereza y cobardía que les impide servirse de su propio entendimiento. El hombre ilustrado, por el contrario, supera la minoría de edad mediante la razón, logrando de esta forma la autodeterminación: "la decisión de alcanzar la libertad, auto-conduciéndose y prescindiendo de la dirección ajena, es un logro de la razón ilustrada, para la cual sólo puede existir la libertad, lo que conduce paulatinamente a la emancipación del género humano. En otras palabras, solo por obra de la razón (por definición un estado de reflexión superior a la intuición y al entendimiento) se obtiene la liberación de las ataduras externas, abriendo paso al gobierno autónomo de las acciones. Por consiguiente, sin Ilustración no es posible la libertad y sin Ilustración tampoco es viable la modernidad" (Robles 2012:171).

La razón del hombre ilustrado, capaz de pensar por sí mismo, se constituye en emblema esperado de la modernidad, al tiempo que, en consecuencia, se problematiza la conformación de una moral ilustrada que orienta la acción a partir de nociones de una vida buena o deseable, que trasciendan las prescripciones de tipo religioso y que se conectan a una fuente universal de legitimidad moral dada por la razón en su uso práctico: "a diferencia del uso teórico de la razón que se ocupa del conocimiento de objetos dados, el uso práctico de la razón tiene que ver con la orientación de la vida humana a partir de la representación de ideas de la razón, por esos sus leyes no determinan lo que es, sino lo que debe ser" (Martínez 2006:188). Estas consideraciones kantianas sobre una moral ilustrada propias de la modernidad, son la base de la aspiración de una sociedad donde sus propios miembros determinan aquella forma de vida que deciden vivir. Esto es la promesa de la autodeterminación social (Güell. Subjetividad social). Ahora, dicha aspiración social expresa una concepción particular del tiempo, propio de la racionalidad del mundo moderno "que devela el reemplazo del pasado por el futuro, como dimensión sobre la cual recaen las expectativas de la sociedad" (Quijano 1988:12).

La emancipación del hombre y su promesa de autodeterminación, situada en el contexto de una conciencia histórica, ubicada temporalmente en el marco de la reflexión intelectual de la ilustración europea, permite configurar un tipo de racionalidad moderna junto a un proceso de producción de identidades/alteridades donde Europa, simultáneamente, se autodefinirá e inventará a sus otros, fundamentalmente como seres inferiores (Duchet. Antropología e historia en el siglo de las luces). En tal sentido, la noción de desarrollo como discurso y modelo de acción geopolítica se instala en terreno fértil. Las bases de la modernidad proporcionan al desarrollo el contexto paradigmático y epistémico que le da sentido. Sin embargo, "las mutaciones introducidas por el advenimiento de la modernidad no están representadas exclusivamente por la conformación de nuevas relaciones intersubjetivas, sino que además están acompañadas por la estructuración de un hasta entonces inédito modelo de control y de explotación del trabajo, que incidirá de manera equivalente en todos los ámbitos de la existencia social" (Quintero 2013:74). 
Esto es el capitalismo que, junto con proporcionar los parámetros de medición del éxito del desarrollo, ha posibilitado la clasificación social entre subdesarrollados/desarrollados, primer/tercer mundo, etc. A la vez ha consolidado modos de vida sustentados en valores como el individualismo, la competencia, la ganancia y el interés propio (Araujo. Sujeto y neoliberalismo en Chile), los cuales se vuelven funcionales a las estrategias de desarrollo que hace ya siete décadas impulsó Truman.

Sin embargo, luego de siglos de modernidad y décadas de intensos programas de desarrollo (bajo diversas modalidades y denominaciones; sustentable, humano, local, etc.), los cuestionamientos a la modernidad como base epistemológica que sustenta la noción de desarrollo se hacen más patentes que nunca. La promesa de autodeterminación social entra en contradicción con la subyugación y formas de explotación que el propio desarrollo posibilitó: "En vez del reino de abundancia prometido por teóricos y políticos de los años cincuenta, el discurso y la estrategia del desarrollo produjeron lo contrario: miseria y subdesarrollo masivos, explotación y opresión sin nombre. La crisis de la deuda, la hambruna, la creciente pobreza, desnutrición y violencia son apenas los síntomas más patéticos del fracaso de cincuenta años de desarrollo" (Escobar 2007:21).

El desarrollo evidencia en este sentido un doble filo, por un lado, releva las capacidades humanas y, por otro, es capaz de "penetrar corazones, almas y de construir una figura subjetiva que pueda funcionar dentro de los dispositivos del capitalismo contemporáneo" (Fagioli 2018:574). Lander nos lleva a reflexionar sobre una crisis civilizatoria: "el patrón civilizatorio antropocéntrico, monocultural y patriarcal, de crecimiento sin fin y de guerra sistémica que hacen posible la vida en el planeta tierra, atraviesan una crisis terminal. La civilización de dominio científico-tecnológico sobre la llamada 'Naturaleza', que identifica el bienestar humano con la acumulación de objetos materiales y con el crecimiento económico sin medida -cuya máxima expresión histórica es el capitalismo- tiene el tiempo contado. Su dinámica destructora, de mercantilización de todas las dimensiones de la vida, socava aceleradamente, las condiciones que la hacen posible" (Lander 2014:27).

Lo que en definitiva se ha quebrado es la conciencia de nuestro entendimiento respecto de quienes somos y el lugar que ocupamos en el mundo, un quiebre en la forma de entender el progreso y las formas de conocimiento asociadas a éste, en tal sentido es un quiebre también que nos devela que la autodeterminación social prometida por la modernidad no solo no es la única forma de concebir el devenir de la humanidad, sino que éste mismo nos ha llevado a caminos que justifican la explotación, abuso y jerarquización de grupos humanos y la propia naturaleza.

Bajo esta mirada, la reflexión se sitúa en una adecuación de la tradición de la crítica social que Sembler vincula a una teoría de la reproducción social: "en efecto, la necesidad de describir los modos en que se reproduce el orden social se vincula no solo a su crucial tarea de describir las distintas distorsiones o patologías que su reproducción experimenta en el contexto histórico del capitalismo, sino además permite situar a la propia crítica como un momento de autorreflexión de una praxis socialmente existente cuyo potencial racional puede servir a los fines de una transformación. Los fines emancipadores de la crítica social son vistos, de esta manera, no como abstracciones o simples ideales perseguidos por el investigador, sino en tanto componentes de una praxis racional que resulta crucial para entender la reproducción del orden social" (Sembler 2018:378).

Las evidentes paradojas de la modernidad nos plantean la necesidad de volver a interrogar filosóficamente el concepto de desarrollo, desde presupuestos que enaltezcan las capacidades humanas y que de algún modo nos devuelvan la promesa de la autodeterminación social centrada en la respuesta al quiénes 
somos. Considerando esto, se discutirá la noción de desarrollo a partir del segundo estudio de la obra Caminos del reconocimiento de Paul Ricoeur, estableciendo un diálogo que permita reubicar epistemológicamente las capacidades individuales y las prácticas sociales como los fundamentos para lograr un desarrollo que apueste hacia el reconocimiento mutuo, sustentado desde lo ético, en la autodeterminación y la justicia social.

\section{Sobre el reconocimiento y sus caminos}

Ricoeur parte su reflexión filosófica a partir de un análisis lexicográfico de la palabra reconocer. Plantea un trastocamiento, tanto en el plano gramatical como filosófico, proponiendo a modo de hipótesis un ordenamiento de los usos filosóficos potenciales que irían desde lo que él denomina voz activa a la voz pasiva. En el primer caso, uso en cuanto a verbo señala: "reconocer algo, objetos, personas, así, a otros, al uno al otro y en el segundo caso, en voz pasiva a ser reconocido, pedir ser reconocido" (Ricoeur 2006:33). Este cambio de voz activa a pasiva implica, según el autor, el superar la dimensión cognoscitiva del reconocer hasta llegar a un plano social del reconocimiento, que implique a los otros sujetos que componen la alteridad vía reciprocidad. Ahora bien, los caminos propuestos por el autor son los siguientes.

El primer camino es el reconocimiento como identificación, el cual está enfocado en la relación sujeto objeto, al cómo el hombre distingue y reconoce objetos, personas y cosas. Este camino se queda en el plano del conocimiento, o en voz activa como señala Ricoeur. El segundo camino plantea el reconocerse a sí mismo, es decir la mirada se vuelve hacia el hombre, particularmente hacia la pregunta ¿quién soy?, la fenomenología del hombre capaz y la hermenéutica del sí mismo. La constitución del yo en base a la alteridad y el reconocimiento vía atestación. Se analizan las capacidades individuales y luego las colectivas como elemento de transición hacia el último camino del reconocimiento mutuo. El tercer camino aborda el reconocimiento mutuo, donde se visualiza muchas veces a la alteridad como una dificultad u obstáculo para el desarrollo de las propias capacidades, se abre el camino entonces de la lucha y la reivindicación por ser reconocido. Lo que el autor llama voz pasiva.

Para el desarrollo de la problemática antes descrita, se considerará específicamente los planteamientos respecto del hombre capaz y el desarrollo de las capacidades colectivas, ambos vistos desde esta perspectiva filosófica como el puente que permitiría transitar hacia el camino del reconocimiento mutuo, y que desde el plano de la teoría social otorgarían los fundamentos para pensar una noción de desarrollo bajo un enfoque ético orientado al bien común, al reencuentro social, centrado en el sujeto y su subjetividad.

\section{El camino del reconocerse a sí mismo: de las capacidades individuales a las capacidades sociales}

El reconocerse a sí mismo implica, según Ricoeur, la existencia de una conciencia reflexiva en el sujeto, que a juicio del autor es lo que distinguiría fundamentalmente el obrar de hombres modernos con respecto a la forma de obrar del mundo griego. El ¿quién soy? constituirá entonces una pregunta constante en el existir humano.

A través de la fenomenología del hombre capaz, Ricoeur establece las dimensiones que sustentan las capacidades individuales en base a la reflexividad y abren el camino del reconocimiento del sí mismo, lo que se desarrolla en cuatro ámbitos: (a) En la acción. En que el sí mismo se designa como autor de una acción que depende de sí, por lo que el sujeto puede reconocerse como agente y con capacidad de obrar. (b) En los actos de habla. En que el sí mismo se designa como hablante, como emisor de enunciados. Se 
destaca que el ser humano es lenguaje, se constituye e interpreta a través de él, siendo el principal medio para relacionarnos con el mundo, con los otros y nosotros mismos. (c) En el poder contar y poder contarse. Con la capacidad de narrar y narrarse mediante historias y configurar así una identidad propia, mediante la elaboración de tramas y construcción de personajes. (d) En la imputación moral. En que el sí mismo se designa como sujeto responsable o imputable de sus acciones.

Es relevante mencionar que estas capacidades humanas no son reconocidas a modo de certezas epistémicas, sino que son reforzadas vía atestación, como forma de seguridad y confianza de seguir siendo los mismos: "la atestación define el tipo de certeza a la que puede aspirar la hermenéutica no solo respecto a la exaltación epistémica del cogito planteada por Descartes, sino que también a su humillación en Nietzche" (Ricouer 2006:xxxiv). De este modo, atestación "se opone principalmente a la noción de episteme, de ciencia, de saber último autofundante" (Ricoeur 2006:xxxv), sino que nos remite a "la seguridad de ser uno mismo agente y paciente" (Ricoeur 2006:xxxvi). Se refuerza así la importancia de la sociedad como el otro generalizado que legitima la identidad personal de los sujetos. La identidad del sí mismo no se forma sin el diálogo con el otro.

El sujeto pedirá ser reconocido como un sí mismo vía estas capacidades, que se constituirán para Ricoeur en el camino hacia el reconocimiento mutuo. El paso de las capacidades individuales hacia la atestación de las capacidades sociales. En el ejercicio de estas capacidades, el hombre manifiesta y construye su identidad personal, imbricado con el otro, en un permanente proceso de constitución en respuesta a la interacción social. Lo relevante es que Ricoeur considera al individuo como un hombre capaz que en su condición de agente puede expresar dichas capacidades en el plano social, transitando desde el camino del quién soy hacia el reconocimiento mutuo.

\section{Capacidades y prácticas sociales como puente para el reconocimiento mutuo}

En el segundo estudio sobre el camino del reconocimiento de sí, Ricoeur ensancha el análisis de las capacidades individuales hacia las capacidades sociales, bajo el entendido que esto constituiría el puente hacia el camino del reconocimiento mutuo, el cual es presentado en su tercer estudio. A decir del autor: "en esta acepción ampliada las capacidades en cuestión no solo son atestadas por individuos sino también por colectividades y sometidas a la apreciación y aprobación pública” (Ricoeur 2006:173).

¿Pero qué es lo que modificaría esta modalidad de reconocimiento y cómo? Ricoeur siguiendo a Amartya Sen, plantea que se trataría fundamentalmente del poder de obrar, de desarrollar capacidades en base a derechos, a la capacidad de agenciación de los sujetos. Señala este autor un fuerte y claro vínculo entre las nociones de representaciones colectivas y prácticas sociales: "La idea de capacidades encuentra su justificación en el acoplamiento entre representaciones colectivas y prácticas sociales, la esfera de las representaciones realiza la función de mediador simbólico y lleva al primer plano la cuestión de la identidad de las entidades sociales en juego. El campo de las prácticas sociales remite al lugar de honor al agente del cambio, el protagonista social" (Ricoeur 2006:176).

Se evidencia así un vínculo entre las representaciones colectivas, como mediaciones simbólicas, que confieren identidad a los vínculos sociales. Ahora bien, el realizador de las prácticas sociales interpela tanto al individuo como a la colectividad, en cuanto a su capacidad de obrar y rol como agentes de cambio. Sen reafirma que "son los propios individuos los que deben asumir la responsabilidad del desarrollo y de la transformación del mundo en que viven" (Sen 2000:338). Sin embargo, dicho enfoque supone que las capacidades deben ser equivalentes a derechos. Plantea Ricoeur: "aquí es donde interviene la libertad de 
la que dispone el individuo y, con ella, la cuestión de los derechos que transforman a las libertades abstractas en oportunidades reales" (Ricoeur 2006:182). Así, finalmente la libertad individual de elección de una vida deviene en una responsabilidad social.

Concepción de responsabilidad social que hace de la libertad individual el objetivo primero de la teoría de la justicia. La libertad se convierte en elementos de evaluación de los sistemas sociales, incluso en su dimensión económica. El "derecho a ciertas capacidades de obrar" asume un supuesto que vincula la "libertad" como condición de posibilidad de elegibilidad, bajo la modalidad de "evaluación de las situaciones" donde toma un protagonismo la responsabilidad colectiva, en definitiva, se trata de una paradójica vinculación entre libertad y responsabilidad colectiva.

Para Cejudo "la idea de libertad como capacidad estará incompleta mientras no se inscriba en las teorías Senianas de la elección social, los derechos y el desarrollo. Solo bajo esta perspectiva más amplia se puede evaluar su pretensión de ser un concepto universalizable de libertad, apto para las sociedades pluriculturales y evaluable con algún tipo de índice de desarrollo humano (Cejudo 2007:17). Por lo tanto, y a partir de la teoría Seniana de la elección social, Cejudo señala que "las capacidades inciden especialmente en la dimensión positiva de la libertad por ser un tipo de poder" (2007:18). Para Cejudo el enfoque de las capacidades son, en definitiva, una forma de entender la libertad y para Sen "la libertad es un fin en sí mismo del desarrollo" (Sen 1999:24). El sujeto participa como protagonista de su propio destino, dirá Sen. La libertad real de oportunidades se halla en él.

El enfoque seniano se inclina por lo que los individuos pueden hacer realmente con los bienes. La pluralidad en esta propuesta de Sen se condice con la exigencia de la libertad en el logro mismo, donde el sentido de justicia se reconcilia con la autonomía de la voluntad de cada individuo como pluralidad (Jiménez Castillo. Una arqueología hegeliana de las teorías del desarrollo). Se deriva así de la propuesta seniana seguida por Ricoeur el énfasis en la capacidad de obrar por sobre las interferencias abusivas del sistema social. Ahora, eso no significa que la capacidad de obrar se realice sin consideración de la libertad garantizada por las instituciones políticas y jurídicas, por el contrario, en este sentido, plantea Ricoeur, "el reconocimiento y atestación cede su sitio a formas de justificación ético jurídicas que cuestionan la idea de justicia social" (Ricoeur 2006:173). Para Ricoeur, se trataría de una "concepción de obrar humano enraizado en la antropología fundamental, en este nivel donde se justifica la convergencia entre el binomio que une representaciones y prácticas sociales y el de derechos a capacidades" (2006:187).

Ahora bien, para desplegar el enfoque de las capacidades se requiere para Sen estar habilitado, que define como los recursos bajo el poder del sujeto. Así, "los enfoques de las capacidades y las habilitaciones son complementarios, ya que las primeras son el marco normativo en el que afrontar la distribución de las habilitaciones, mientras que éstas son una variable en la estimación del bienestar mediante capacidades" (Cejudo 2007:12).

Como sea, la idea de sujeto supone una conciencia libre, que, tal como se señaló en el primer punto de este ensayo, en el marco de la modernidad, implica desplegar la capacidad de autodeterminación desde lo individual a lo social. En tal sentido el sujeto se vincula a la capacidad de obrar para lograr el ideario indicado de autodeterminación. Según Ricoeur: "la idea de poder obrar, de agency, en el que opera la evaluación de nuestras capacidades, unida de modo secreto a la idea de vivir bien" (2006:189). De este modo, el camino hacia el reconocimiento mutuo supone un quiasmo entre libertad y capacidad: el hombre capaz en Ricoeur y el hombre habilitado en Sen reflejan la síntesis para el despliegue de las capacidades 
sociales en la búsqueda del bienestar, donde las capacidades personales requieren de habilitaciones sociales, que estén por cierto enraizadas en una profunda sensibilidad ética e institucional.

\section{Desde la modernidad a Ricoeur: el desarrollo como una intencionalidad ética}

A partir de los planteamientos filosóficos de Ricoeur, en torno a la constitución del sujeto, de sus capacidades de obrar de forma individual y social, podemos concluir con algunas claves dialogantes entre la nociones de desarrollo y de reconocimiento, que por cierto compatibilizan con definiciones inscritas en la modernidad (desarrollo humano y post desarrollo, por ejemplo), pero que además nos ayudarán a estructurar respuestas a las interrogantes éticas asociadas al bienestar, como fin último del desarrollo. El hombre capaz es, sin duda, el comienzo para entender los vínculos entre capacidades individuales y sociales. Desde lo singular a lo grupal, como es muy bien reflejado en Los caminos del reconocimiento a modo de escalas, siempre apoyándose en la idea de las capacidades senianas.

El punto articulador entre la apuesta ricoeuriana y la noción de desarrollo estaría dada de forma general por el tránsito del camino del reconocimiento de sí mismo hacia el reconocimiento mutuo, conexión que además está mediatizada por su reflexión ética. Dice Ricoeur: "Ilamemos intencionalidad ética al deseo de una vida buena, con y para los otros, en instituciones justas" (1996:176). Para este autor, primero un claro interés y supremacía del concepto de ética por sobre el de moral: "la ética como intencionalidad de una vida realizada, y moral como la articulación de esa intencionalidad dentro de normas caracterizadas por la pretensión de universalidad y por un efecto de restricción (1996:174). La primera vista entonces como deseo o anhelo y la segunda como norma coaccionante que demanda obligatoreidad. Ahora bien, de la definición dada, tres son los elementos constitutivos de la intencionalidad ética propuesta por Ricoeur. Así mismo tres serán los imperativos dialogantes con la noción de desarrollo.

Tender a la vida buena. Señala Ricoeur: "vida buena objeto mismo de la intencionalidad de la ética cualquiera sea la imagen que cada uno se hace una vida realizada" (1996:177). De esta forma se apela a lo que cada individuo responsablemente elige para su plan de vida en base a sus deseos y anhelos, que Ricoeur define como "nebulosa de ideales y de sueños de realización, respecto al cual una vida es considerada realizada o más o menos realizada". Es decir, no existe una norma que establezca qué es la vida buena, sino que simplemente obedece a lo que cada uno aspira a construir.

De lo anterior, dos ideas fundamentales: en primer lugar, la capacidad de elegir por razones, de preferir esto a lo otro, esto es, la capacidad de actuar intencionalmente. Lo segundo, la capacidad de introducir cambios en el curso de las cosas, de comenzar algo en el mundo. Dice Ricoeur que "lo estimable en el sí mismo son las capacidades de elegir y de introducir cambios en la vida, porque de ese modo el sí mismo estructura su vida mediante su praxis, sus propias acciones y elecciones" (2002:234). Entonces, si pensamos ¿quién es el sujeto de desarrollo? y ¿quién define ese bienestar?, claramente es un sí mismo con capacidad para estimar, valorar, reconocer y elegir su camino de desarrollo, en tanto que posee las capacidades individuales para obrar y decidir su vida. El sujeto entonces como agente y protagonista del cambio social.

Con y para el otro. El encuentro con el otro denominado por Ricoeur como solicitud, implica la idea de reciprocidad en las relaciones sociales, que por un lado nos permiten reconocernos como capaces y viceversa. Plantea: "los agentes y pacientes son tomados en relaciones de intercambio que, como el lenguaje, conjugan reversibilidad de las funciones e insustituibilidad de las personas, lo que la solicitud añade es la dimensión de valor que hace que cada persona sea irremplazable en nuestro afecto y nuestra 
estima" (Ricoeur 1996:201). De algún modo el reconocimiento de sí mismo, vía atestación, permite identificar al otro como sujeto con capacidades de obrar colectiva e individualmente: "Como a mí mismo significa, tú también eres capaz de comenzar algo en el mundo, de actuar por razones, de jerarquizar preferencias, de estimar los fines de tu acción" (Ricoeur 2006:202). De esta forma, la vida buena no queda encerrada en uno mismo, sino que se realiza con y para los otros. Según Ricoeur: "la solicitud no se añade desde fuera a la estima de sí, sino que despliega la dimensión dialogal implícita en ella. Estima de sí y solicitud no pueden vivirse y pensarse una sin la otra. Decir sí no es decir yo. El sí implica ya lo otro, si es que se puede decir de alguien que se estima a sí mismo como a otro" (Ricoeur 2002:243).

En este sentido, el sujeto no solo es responsable por sí mismo, sino también es responsable del otro. Señala Ricoeur: "la amistad no solo incumbe realmente a la ética, como la primera manifestación del deseo de vivir bien; sino, sobre todo, lleva al primer plano la problemática de la reciprocidad" (1996:189). Es decir, la reciprocidad se impone en el plano ético de la amistad, porque supone que cada uno desea el bien para el amigo: "a la estima de sí, la amistad añade sin quitar nada. Lo que añade es la idea de reciprocidad en el intercambio entre humanos que se estiman mutuamente" (Ricoeur 1996:195).

La presencia de la reciprocidad evidencia también la existencia de una relación desigual donde el otro es un ser sufriente, que ha perdido su capacidad de obrar o sus habilitaciones para hacer. Es por ello por lo que la solicitud se encargará de restablecer el orden en esa relación desigual. Para Ricoeur la reciprocidad demanda reconocer al otro como un ser insustituible, como un ser único e irremplazable. De ese modo, la estima de sí se amplía hacia el otro en la dinámica del reconocimiento mutuo. Así entonces el bienestar de una vida buena no se consigue de forma individual, pues en el bienestar del sí mismo ya está incorporado el bienestar del otro.

En instituciones justas. El deseo de una vida buena para Ricoeur se extiende a las instituciones y éstas como punto de partida para la aplicación de justicia en un sentido ético. Por institución entenderá "la estructura del vivir bien juntos de una comunidad histórica, por costumbre comunes y no por reglas coaccionantes" (Ricoeur 2006:203). De nuevo figura la idea de deseo por sobre el de la norma: se apela a la organización institucional en base al consenso social y a la mediación simbólica. ¿Pero qué es lo justo según Ricoeur? Comprende la extensión de las relaciones interpersonales en las instituciones. La noción de igualdad y de garantizar a cada persona su derecho estará a la base de esta noción de justicia: "La igualdad es la vida en las instituciones, lo que la solicitud a las relaciones interpersonales... la justicia acrecienta la solicitud, que en cuanto que el campo de aplicación de la igualdad es toda la humanidad" (Ricoeur 2006:212).

De acuerdo con Ricoeur esto supone ampliar la noción del otro, desde el tú al otro desconocido: "El otro es también otro que tú. Correlativamente, la justicia va más allá del cara a cara" (2002:244). Señala que la justicia se define por una operación distributiva que involucra a la comunidad política: "La justicia consiste precisamente en dar a cada uno lo suyo. El cada uno es el destinatario de un reparto justo" (2002:245). Entonces, ¿cómo lograr ese objetivo de tender a la vida buena con y para el otro? A la base de estos planteamientos se vislumbraría una noción de desarrollo que no solo asume las capacidades individuales y sociales, sino que también promueva el despliegue de capacidades a niveles institucionales que habiliten a los individuos mediante oportunidades para ejercer la libertad para escoger una vida buena. La perspectiva ética propuesta por Ricoeur se articula en torno a la triada: sí mismo, el otro y la justicia, todos como elementos constituyentes de la vida buena: la justicia incrementa la solicitud por el otro, mostrando que la realización personal siempre incluye a cualquiera de esos otros que son iguales al sí mismo. Finalmente, mediante la relación con los otros se fundamentará éticamente la vida humana. 


\section{Conclusiones}

La acción ideológica y burocrática vinculada al poder estatal para implementar una noción de desarrollo coherente con una racionalidad moderna en crisis, es objeto de reflexión a partir de un debate articulado en un nivel epistemológico afín a las interrogantes éticas interpeladas en la propia idea de desarrollo, en tal sentido, el camino al reconocimiento mutuo permite visualizar una noción de desarrollo sustentado sobre un sustrato ético que se definiría según los siguientes aspectos.

Que se reconozca al sujeto como un sí mismo capaz de obrar y de decidir responsablemente sus acciones de vida para su propio bienestar. Noción de desarrollo que implica un diálogo con las capacidades y elecciones que realiza el sujeto. Que las acciones individuales se puedan insertar en proyectos colectivos y puedan ser reconocidas como capacidades sociales. Noción de desarrollo que implica un bienestar que se construye mediante la alteridad social. Que a la base de ese agente y de esa colectividad existe un orden simbólico determinado que precisa ser reconocido. Noción de desarrollo que dialoga con los elementos culturales propios de cada colectividad. Que existe a nivel institucional la disposición de brindar oportunidades y derechos para que cada individuo obre de acuerdo lo estime conveniente, en un marco de justicia, igualdad y respeto por la diversidad. Noción de desarrollo con énfasis en lo estructural y la pluralidad.

Para Sen, quien interpreta al desarrollo como un concepto que va más allá del crecimiento y de los niveles de la renta per cápita de un país o de un territorio, lo realmente importante es que las personas lleven a cabo aquellas funciones y actividades que desean y sean capaces de realizar. Es decir, el desarrollo económico se consigue mediante la utilización de las capacidades que las personas han desarrollado gracias a los recursos materiales, humanos y a la cultura que posee el territorio. Ricoeur, si bien no se enfoca en definir una teoría sobre el desarrollo, se podría decir que sus formulaciones éticas en Los caminos del reconocimiento establecen imperativos dialogantes. Dicha conexión nos lleva a plantear niveles de reconciliación entre los conceptos de imparcialidad y pluralismo propuestos por Jiménez Castillo. Tan imparcial como para poder establecer normativamente que desarrollo implica libertades, necesidades, capacidades y habilitaciones, y pluralista como para reconocer las distintas sensibilidades de los actores, sí mismos participantes en ella. De este modo, los caminos del reconocimiento iluminan en alguna medida la ruta filosófica del desarrollo en el contexto de la modernidad.

Sin embargo, las opacidades propias de la traducción empírica de la noción de desarrollo nos llevan a preguntarnos: ¿cómo hacemos posible este sueño de la autodeterminación social, cuando las paradojas de la modernidad han venido limitando las capacidades del ser humano, a sus posibilidades de elegir, restringiendo sus acciones autodeterminadas? ¿Nos quedan instancias para poder obrar? ¿El contexto institucional actual nos brinda derechos para actuar libremente? ¿Hay espacio para nuestra subjetividad en este escenario social?

La existencia entonces de contracapacidades apuntaría a aquellas formas propias del entorno social que determinarían negativamente el modo en el que se manifiesta la libertad de agencia para funcionar. Este estado de negatividad como "contracapacidad" nos encamina necesariamente hacia la irrupción en el seno del enfoque seniano de un conflicto de naturaleza normativa entre libertad y el sentido de justicia (Jiménez Castillo. Amartya Sen frente al espejo social de la libertad). Por lo tanto, la modernidad como escenario factual nos estaría brindando contracapacidades más que capacidades, esto es, elementos que, irrumpidos desde el entorno social, restringen nuestra libertad para funcionar y poder escoger una vida buena. 
Al respecto, Norbert Lechner también se pregunta cómo realizar la autonomía individual que la sociedad proclama desde las condiciones subjetivas que esta misma sociedad promueve. El mismo responde: "en definitiva las capacidades de la sociedad de intervenir en su propio desarrollo dependerán de la auto imagen que ella tenga de sí" (Lechner 2002:12). Por lo tanto, señala que es preciso desarrollar un imaginario del nosotros en la medida en que realicemos experiencias exitosas de acción colectiva. En consecuencia, las preguntas serían entonces, luego del quién soy, responder el quiénes somos nosotros. Castoriadis dirá: "somos una colectividad autónoma formada por individuos autónomos, y que podemos observarnos, reconocernos, interrogarnos en y por nuestras obras" (1997:96). Con dicha afirmación, el camino al desarrollo sustentado en una ética de libre elección demandará constantemente de la reflexión individual y social, para auto reconocerse y construir no solo un imaginario social del hombre capaz, sino también el de una sociedad que puede y quiere.

\section{Bibliografía}

Castoriadis, C. (1997). El avance de la insignificancia. Eudeba.

Cejudo, R. (2007). Capacidades y libertad: una aproximación a la teoría de Amartya Sen. Revista Internacional de Sociología 65(47): 9-22. https://doi.org/10.3989/ris.2007.i47.50

Escobar, A. (2007). La invención del tercer mundo. Construcción y deconstrucción del desarrollo. Fundación Editorial El Perro y La Rana.

Fagioli, A. (2018). Acumulación originaria y capitalismo neoliberal. Una posible lectura del Chile postgolpe. Isegoría 59: 573-593. https://doi.org/10.3989/isegoria.2018.059.11

Jiménez-Castillo, M. (2017). Una arqueología hegeliana de las teorías de desarrollo. Cinta moebio 59: 186-197. https://doi.org/10.4067/S0717-554X2017000200186

Martínez, H. (2006). Kant, una ética para la modernidad. Diálogos de Saberes 24: 181-194. https://revistas.unilibre.edu.co/index.php/dialogos/article/view/4307

Lander, E. (2014). Crisis civilizatoria, límites del planeta, asaltos a la democracia y pueblos en resistencia. En: P. Quintero. Crisis civilizatoria, desarrollo y buen vivir (pp. 27-57). Ediciones del Signo.

Lechner, N. (2002). Las sombras del mañana: la dimensión subjetiva de la política. LOM ediciones.

Ricoeur, P. (1996). Sí mismo como otro. Siglo XXI.

Ricoeur, P. (2002). Ética y moral. En: C. Gómez (Ed.) Doce textos fundamentales de la ética del siglo XX (pp. 241-255). Alianza.

Ricoeur, P. (2006). Caminos del reconocimiento: tres estudios. Fondo de Cultura Económica.

Robles, F. (2012). Epistemologías de la modernidad: entre el etnocentrismo, el racionalismo universalista y las alternativas latinoamericanas. Cinta moebio 45: 169-203. https://doi.org/10.4067/S0717554X2012000300001

Quijano, A. (1988). Modernidad, identidad y utopía en América Latina. Sociedad y Política Ediciones.

Quintero, P. (2013). Desarrollo, modernidad y colonialidad. Antropología Experimental 13: 67-83. https://revistaselectronicas.ujaen.es/index.php/rae/article/view/1816

Sembler, C. (2018). Injusticias y emancipación. La renovación de las bases epistemológicas de la crítica social. Cinta moebio 63: 377-390. https://doi.org/10.4067/S0717-554X2018000300377 
Sen, A. (1999). Development as freedom. Oxford University Press.

Sen, A. (2000). La libertad y los fundamentos de la justicia. Desarrollo y libertad. Editorial Planeta.

Recibido el 1 Oct 2019

Aceptado el 26 Dic 2019 